This document is the unedited Author's version of a Submitted Work that was subsequently accepted for publication in J ournal of Physical Chemistry C, copyright (c) American Chemical Society after peer review. To access the final edited and published work see:

https://dx.doi.org/10.1021/acs.jpcc. 9b06516. 


\section{On the Suitability of Raman Spectroscopy to Monitor the Degree of Graphene Functionalization by Diazonium Salts}

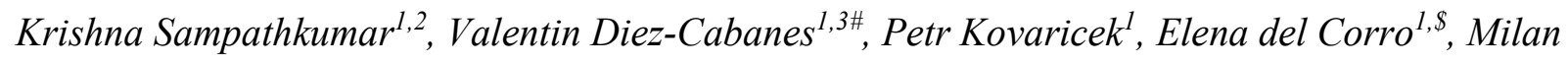
Bouša ${ }^{1}$, Jan Hošek ${ }^{3}$, Martin Kalbac ${ }^{1}$, Otakar Frank ${ }^{1, *}$

${ }^{1}$ J.Heyrovsky Institute of Physical Chemistry of the CAS, v.v.i., Dolejskova 2155/3, 18200 Praha 8, Czech Republic

${ }^{2}$ Central European Institute of Technology (CEITEC), Brno University of Technology, Purkyňova 123, 61200 Brno, Czech Republic

${ }^{3}$ Faculty of Mechanical Engineering, Czech Technical University in Prague, Technická 4, 16 607 Prague 6, Czech Republic

\# current address: Laboratory for Chemistry of Novel Materials, University of Mons, Place du Parc 20, B-7000, Mons, Belgium.

\$ current address: Catalan Institute of Nanoscience and Nanotechnology (ICN2), CSIC and the Barcelona Institute of Science and Technology (BIST), Barcelona, Spain.

AUTHOR INFORMATION

Corresponding Author

* otakar.frank@jh-inst.cas.cz, tel: +420266053446 


\begin{abstract}
Raman spectroscopy is undoubtedly the most frequently used technique for universal characterization of graphene and related materials. Quantification of parameters like disorder or strain is possible through analysis of particular Raman bands. However, under certain conditions, such evaluation can be jeopardized by - sometimes hidden - convolution of more overlapping effects. In this work graphene functionalization by the common nitrobenzene diazonium salt under simultaneous biaxial tensile deformation induced by substrate swelling was investigated by Raman spectroscopy. As expected, the disorder-related D band appeared in the spectra documenting the covalent attack on the graphene lattice. However, the strain-induced shift of the graphene bands exposed additional peaks, masked at exactly the same positions as the unstrained graphene bands. The new bands were assigned to vibrations of the diazonium molecule and its decomposition products adsorbed on top of the functionalized graphene. The external strain thus provided means for more correct quantification of the lattice disorder.
\end{abstract}




\section{Introduction}

Graphene attracts enormous research efforts due to its unique physical properties, such as high carrier mobility, superior thermal conductivity, high optical transparency and extreme mechanical properties. ${ }^{1}$ However, many of graphene's foreseen applications will rely on its precisely controlled electronic structure, modified to meet the needs of the particular utilization. The modifications can span from controlling and fine tuning of the local pools with different charge carrier concentrations, the so-called charge puddles, to altering the band structure altogether and opening a band-gap. Several approaches have been used up-to-date to achieve the latter goal, namely 1D confinement into nanoribbons, ${ }^{2}$ chemical functionalization, ${ }^{3}$ or mechanical strain. ${ }^{4-5}$ Naturally, chemical functionalization of graphene through covalent bonding, ${ }^{6-10}$ i.e. rehybridization from $\mathrm{sp}^{2}$ to $\mathrm{sp}^{3}$ character of the carbon atoms, leads in most cases to decrease in conductivity, and eventually to an insulating state, as in fluorographene. ${ }^{11}$ On the other hand, controlled chemical functionalization of the 2D materials is vital for applications in (electro)catalysis or sensing. ${ }^{12-13}$ The functionalized regions can be simply designed by masking, ${ }^{14}$ but the concentration of the attached molecules on graphene's surface is a complex function of the standard reaction conditions (temperature, reactant concentration, solvents, duration etc.) and the state of graphene itself, as one of the main reactants in the process. ${ }^{15-16}$ Herein, the substrate below graphene seems to play one of the crucial roles through an interwoven effect of charge puddles and local strain fields. ${ }^{17-18}$ The mutually interconnected triangle of strain, electronic structure and functionalization thus represents a difficult puzzle to disentangle. However, when fully understood, these three degrees of freedom give us an immense variety of manipulating the innermost properties of graphene.

Aryl radical addition from diazonium precursors is a classical route for a simple chemical functionalization of graphene (and other $\mathrm{sp}^{2}$ carbon materials). ${ }^{6-10,14,19-22}$ These so-called Meerwein arylation proceeds via an electron transfer from graphene to the diazonium cation, followed by the loss of nitrogen to form the reactive aryl radical, which can consequently attack the graphene lattice. ${ }^{8}$ However, the overall reaction process is not as well understood as it might seem. Apart from the complicated substrate effects, ${ }^{17-18}$ even the same reaction under the apparently same conditions conducted by the same group can yield very different degrees of modification of the graphene lattice, as shown e.g. by Koehler and coworkers in refs ${ }^{23}$ and ${ }^{24}$. 
There might be several reasons for this variation, ranging from the formation of dendritic polymers ${ }^{25}$ to the influence of an impurity layer after lithographic processing. ${ }^{23}$

In this letter, we have investigated the process of functionalization of graphene by 4nitrobenzene diazonium tetrafluoroborate (NBD) under continuous biaxial tension reaching up to $0.7 \%$ of strain. The mechanical deformation was achieved by swelling of the polymeric graphene support (SU8 on poly(methylmetacrylate), PMMA) in methanol:water $(1: 1)$ solution. While no perceptible influence of strain on the functionalization level could be discerned, the straininduced shifts of the Raman bands of graphene revealed additional Raman bands originating from the vibrations of NBD and its decomposition products.

\section{Experimental Methods}

Graphene samples were prepared by mechanical exfoliation from Kish graphite and deposited onto poly(methylmetacrylate) (PMMA) slabs covered with $\sim 500 \mathrm{~nm}$ spin-coated SU8 2000.5 (MicroChem). The samples were soft-baked at $65^{\circ} \mathrm{C}$ for $30 \mathrm{~min}$. For swelling experiments, the samples were immersed in methanol:water solution (1:1) and the evolution of the graphene was monitored by Raman spectroscopy. For tests with the diazonium salts, the samples were immersed in a $10 \mathrm{mM}$ solution of 4-nitrobenzenediazonium tetrafluoroborate (Sigma-Aldrich) in 1:1 methanol:water. Before the Raman measurements, the samples were removed from the reaction bath and washed with the pure solvent mixture.

Raman spectra were measured using LabRAM HR (Horiba) microspectrometer, with laser excitation wavelengths of 488, 633 or $785 \mathrm{~nm}$. If not stated otherwise, the presented results were obtained using $633 \mathrm{nmn}$ excitation. $600 \mathrm{l} / \mathrm{mm}$ grating was used, providing $1.8 \mathrm{~cm}^{-1}$ point-to-point spectral resolution for $633 \mathrm{~nm}$ excitation. Typical accumulation time was 40 seconds for one spectral window in the mapping procedures, and 60 seconds in single-spot measurements. For peak analysis, first, the spectra were divided to two parts $\left(1200-1800 \mathrm{~cm}^{-1}\right.$ and $\left.2400-2700 \mathrm{~cm}^{-1}\right)$, followed by subtracting a linear background and normalizing to the intensity of the PMMA $\sim 1450 \mathrm{~cm}^{-1}$ band. After that, pure polymer spectra, measured at the start of the experiment, were subtracted. The peaks were then fitted with Lorentzian lineshapes. AFM images of the graphene with the deposit layer were measured in the tapping mode with a Dimension Icon microscope 
(Bruker Inc.). Images were captured using Bruker SCANASYST-AIR probes $\left(\mathrm{k}=0.4 \mathrm{~N} / \mathrm{m}, f_{0}\right.$ $=70 \mathrm{kHz}$, nominal tip radius $=2 \mathrm{~nm}$ ),

\section{Results and Discussion}

The evolution of graphene with immersion time of the graphene-SU8-PMMA stack is depicted in Figure 1. Raman G and 2D band were monitored to assess the strain and/or potential charge doping levels. During the experiments, no D band appearance was observed indicating no defects were caused by the treatment. Typical Raman spectra before data treatment (for details, see the Experimental details in Supporting information) are plotted in Figure S1 (Supporting Information). Optical image of one of the monolayer graphene flakes is shown in Fig. 1d with the drawn rectangle marking the area where Raman mapping was conducted. Figure $1 \mathrm{f}$ shows the correlation plot of the $\mathrm{G}$ and 2D band frequencies, which is used to separate and potentially quantify the effects of strain and doping. ${ }^{26-27}$ Most of the data points fall onto lines with fitted slopes of $1.52 \pm 0.24(0 \mathrm{~min}), 2.20 \pm 0.07(10 \mathrm{~min})$ and $2.32 \pm 0.04(20 \mathrm{~min})$. At $0 \mathrm{~min}$, i.e. before the start of the experiment, the slope of 1.52 indicates heterogeneity in the flake caused by both strain and doping. With the soaking, the spread of the points increases towards lower wavenumbers both for $\mathrm{G}$ and 2D band and is largely dominated by effects of strain - the slope for biaxial strain is in the range of 2.2 to $2.45 .^{26,28-29}$ As a control experiment, graphene monolayer on $\mathrm{Si} / \mathrm{SiO}_{2}(300 \mathrm{~nm})$ substrate was immersed for a prolonged time (100 $\left.\mathrm{min}\right)$ in the same solvent mixture. Figure S2 (Supp. Info) shows the evolution of selected G and 2D fitted parameters. Apart from minor variations $\left(2-3 \mathrm{~cm}^{-1}\right.$ over the $\left.100 \mathrm{~min} \mathrm{span}\right)$ in band positions as well as linewidths, no significant changes were observed.

The strain level was quantified using the $2 \mathrm{D}$ band shift, with the recent reported rate for polymer-supported graphene of $-148.2 \mathrm{~cm}^{-1} / \%{ }^{28}$ Apart from the highest achieved strain levels around $0.7 \%$, the attained tension was confirmed using the $\mathrm{G}$ band shift. At the highest strain levels, the $\mathrm{G}$ band is sometimes shadowed by the intense bands of the polymer substrate. The maps and profiles of the induced tension (Fig. 1a-c and e) demonstrate the stress being built up from the edges towards the flake's interior. This is reminiscent of the behavior of graphene loaded via beam bending experiments, where the interfacial stress transfer increases from the edge as well and follows the shear-lag theory. ${ }^{30-32}$ Another example is shown in Figure S3 (Supp. 
Info). We propose the biaxial strain is caused by swelling of SU8, which is a known

phenomenon, and can reach high values depending on the post-deposition treatment - the more crosslinked, the less swelling can be induced..$^{33}$ In our case, the relatively low soft-baking temperature allows for SU8 volume expansion of at least $0.7 \%$ (the strain level reached in graphene).
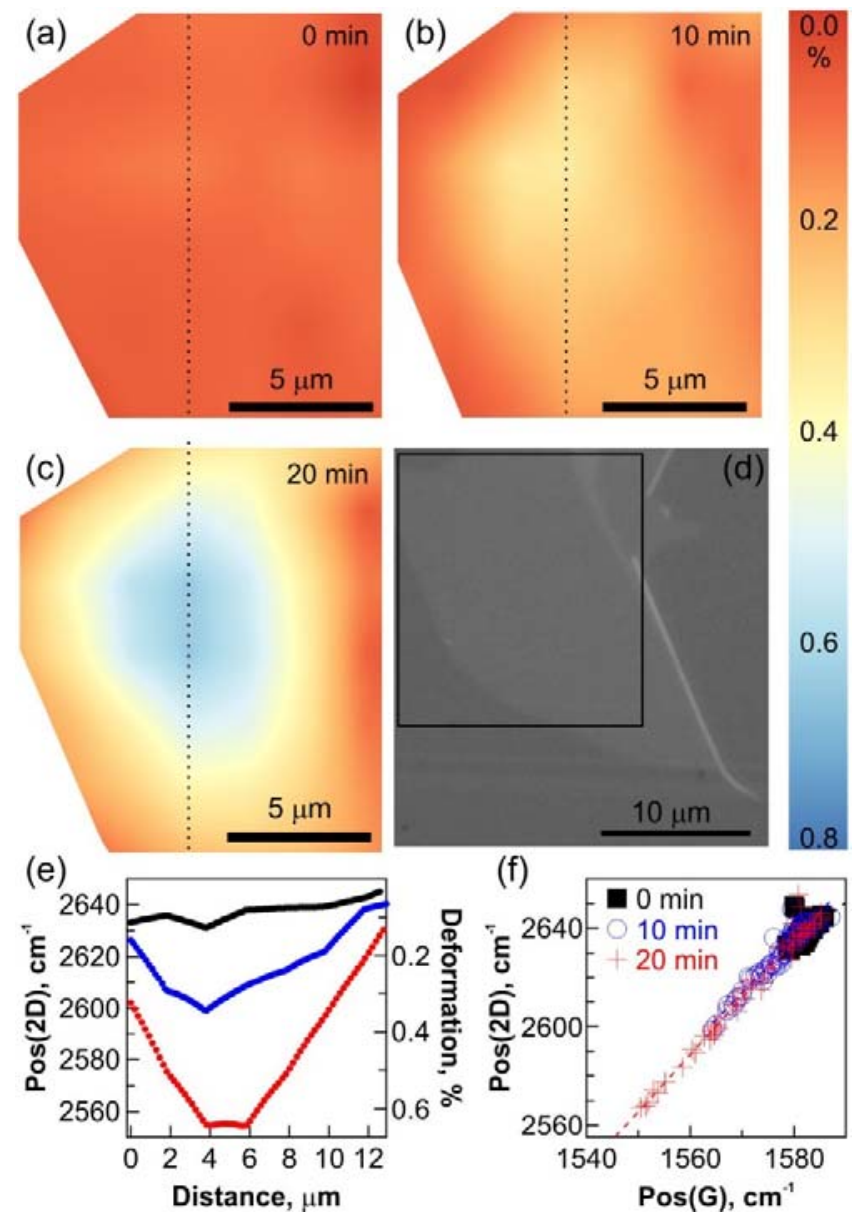

Figure 1. Evolution of the Raman spectral features of monolayer graphene on PMMA/SU8 substrate subjected to swelling in methanol:water solution. (a-c) maps of biaxial strain quantified from the 2D band shift at 0,10 and 20 minutes of soaking. (d) optical image of the flake with the black rectangle marking the mapped area shown in a-c. (e) 2D band shift (left axis) and biaxial strain (right axis) profiles extracted along vertical dashed lines in a-c. (f) correlation of $2 \mathrm{D}$ and $\mathrm{G}$ band frequencies for 0,10 and 20 min soaking. The color coding in e and $\mathrm{f}$ is the same: black -0 min, blue $-10 \mathrm{~min}$, red $-20 \mathrm{~min}$. The mapping step was $2 \mu \mathrm{m}$ in each direction.

For the reactivity experiment, the samples were prepared and monitored in the same way, only $10 \mathrm{mM}$ 4-nitrobenzenediazonium tetrafluoroborate solution in the same solvent mixture was 
employed. Figure 2 shows treated Raman spectra of graphene at 0, 90, 180 and 240 minutes (the raw spectra are plotted in Figure S4, Supporting Information). As can be seen, the Raman G and 2D bands were downshifting as in the case of the 'pure swelling', however, additional bands appeared in the spectra. Most notably a broad feature emerges between 1300 and $1340 \mathrm{~cm}^{-1}$, which, especially at shorter time intervals from the start (cf. 90 min in Fig. 2), could be tentatively assigned as the $\mathrm{D}$ band caused by the progressive bonding of the aryl radical onto the graphene lattice. ${ }^{8}$ The extent of the functionalization would in such a case be assessed by the $\mathrm{D} / \mathrm{G}$ intensity ratio, as is common. ${ }^{34}$ However, as the experiment progresses, so does the swelling of the supporting polymer and the graphene's lattice is expanding along. The deformation causes the $\mathrm{D}$ band to shift at approximately half the shift rate of the $2 \mathrm{D}$ band. As the $\mathrm{D}$ band moves to lower wavenumbers, an additional band located very close to its theoretical original position at $\sim 1330 \mathrm{~cm}^{-1}$ is revealed, increasing in intensity with time. The frequency of the additional band is not changing over time. In other words, the band does not originate directly from the vibrations of the graphene lattice. Similarly, another band appeared at $\sim 1600 \mathrm{~cm}^{-1}$, clearly revealed by the downshifting $\mathrm{G}$ band.

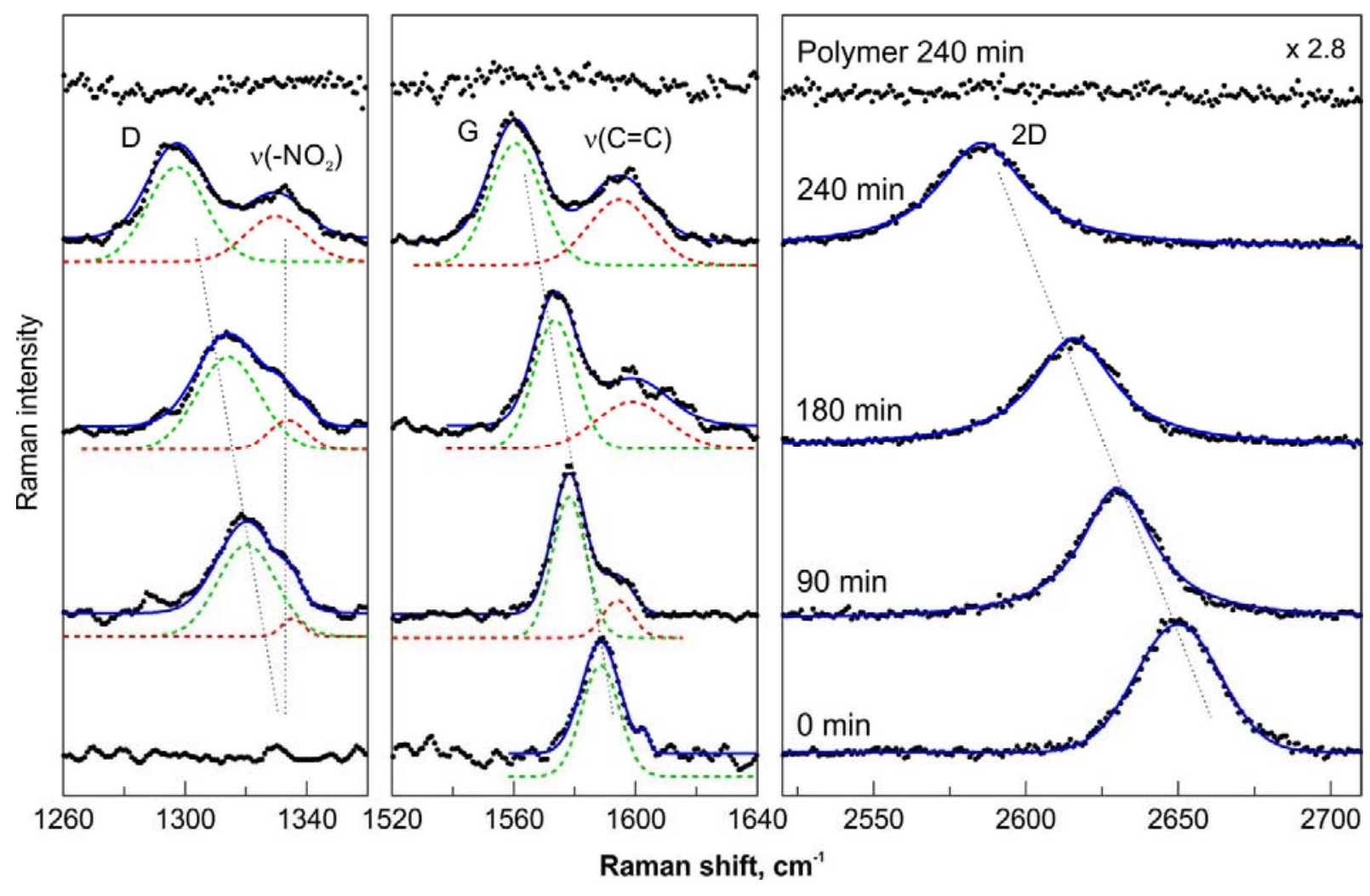


Figure 2. Examples of spectra of monolayer graphene treated with $10 \mathrm{mM}$ NBD in methanol:water 1:1 solution for an indicated time, measured in the approximate center of the flake. The original spectra are plotted as black point, individual Lorentzian shapes as green or red dashed lines and their sum as solid blue lines. The Raman intensity of the 2D band was divided by 2.8 for better comparison. The top curve is the differential spectrum of the treated polymer without graphene measured at 240 and 0 minutes, as an experimental control.
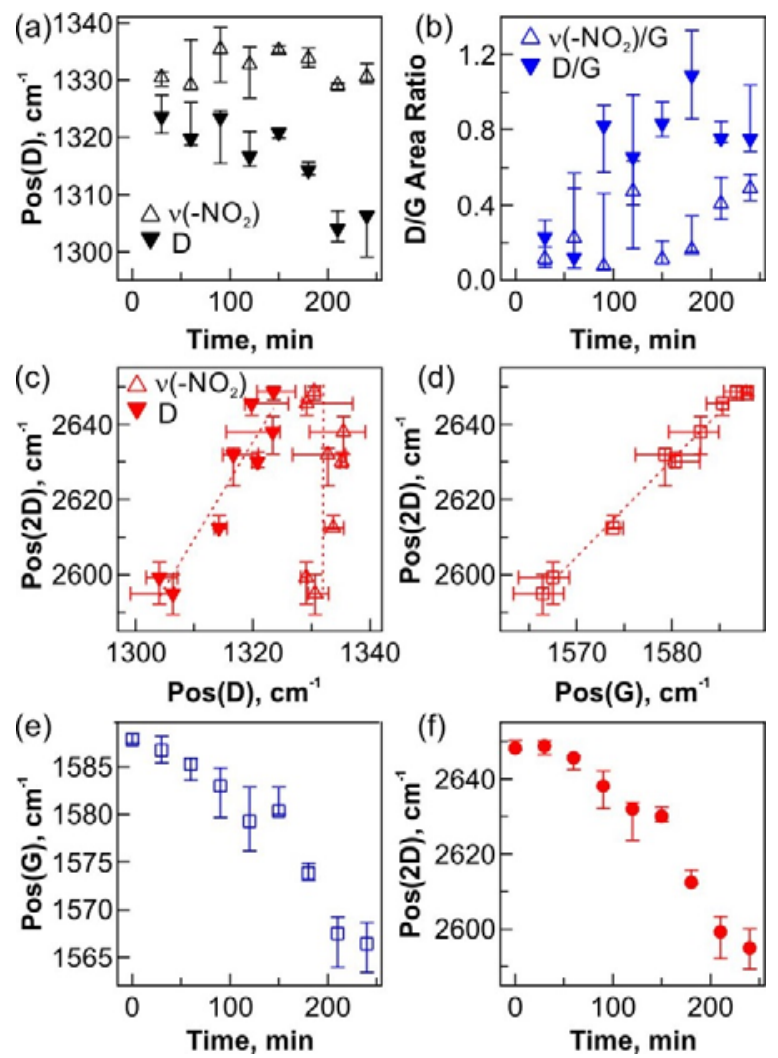

Figure 3. Evolution of selected parameters of the peaks fitted to the Raman spectra of graphene treated with $10 \mathrm{mM}$ NBD in methanol:water. The error bars are the $1^{\text {st }}$ and $3^{\text {rd }}$ quartile of the fitted values, the symbol being the median of 9 measurements at different spots at the sample, acquired at approximately regular steps along a line profile across the center of the flake.

Figure 3 depicts various parameters of the fitted bands. We note that the deconvolution of the ' $D$ band envelope' into two bands is very inaccurate in the first half of the experiment (till $\sim 120$ min) because of (i) the noise in the spectra, and (ii) proximity and large width of the two components. Nevertheless, thanks to the number of measurements, the statistical evaluation 
shows clear trends in the behavior of bands also in the early stages. The real D band downshifts with time, while the unknown band remains stable (Fig. 3a and c). The D band seems to appear quickly and its intensity is stable afterwards, while the unknown band's intensity increases steadily with time (Fig. 3b). There might be an initial influence of doping on the Raman shift of the $\mathrm{G}$ and 2D band, $c f$. the evolution of the first three data points in Fig. $3 \mathrm{e}$ and $\mathrm{f}$, but the main cause of the $G$ and 2D band shifts is the strain. The correlation of the 2D and G frequencies (Fig. $3 d$ ) shows the slope of $2.57 \pm 0.09$. Only at the start of the experiment (data in the top right corner of Fig. 3d) the evolution of the $2 \mathrm{D}$ and $\mathrm{G}$ frequencies reflects the changes caused by the covalent bonding of the molecule.

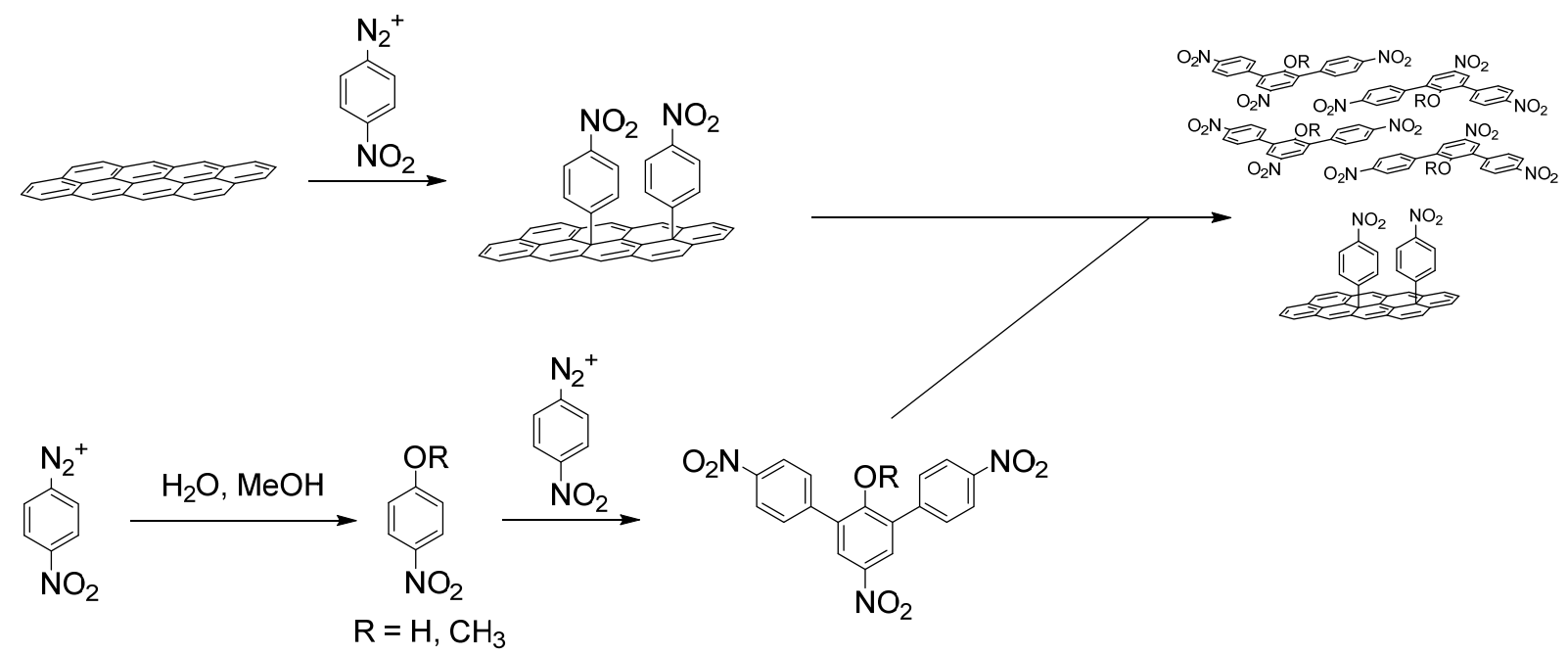

Figure 4. Possible pathways in prolonged NBD reaction with graphene in methanol:water mixture.

The origin of the bands should be looked for in the NBD molecule. The most probable assignment for the $\sim 1330 \mathrm{~cm}^{-1}$ band is the symmetric stretching vibration of the nitro group, $v(-$ $\mathrm{NO}_{2}$ ), which is known to be very strong and between $1320-1360 \mathrm{~cm}^{-1}$ when on an aromatic ring. ${ }^{35}$ The band at $\sim 1600 \mathrm{~cm}^{-1}$ is most likely the $\mathrm{C}=\mathrm{C}$ stretching vibration, $v(\mathrm{C}=\mathrm{C})$, of the aromatic ring in NBD or its decomposition products. Both band assignments have been verified by means of Density Functional Theory (DFT) calculations (see Section 2 in the Supporting Information). The possibility of substrate reactivity was considered but experimentally ruled out through measurements of the polymer next to the studied graphene flakes. The top curves in Fig. 
2 are differential spectra between the polymer after $240 \mathrm{~min}$ in the reaction mixture and the polymer at time zero - only noise can be discerned in the spectra. Given the stagnant intensity of the $\mathrm{D}$ band and the increasing intensity of the $v\left(-\mathrm{NO}_{2}\right)$ and $v(\mathrm{C}=\mathrm{C})$ bands, we can propose a scenario depicted in Figure 4. The reaction between NBD and graphene is typically very fast, reaching a steady state in the order of minutes. ${ }^{24,36-38}$ However, NBD is slowly decomposing in polar protic media, ${ }^{39}$ such as in our case the methanol:water mixture, forming the corresponding 4-nitrophenol or 4-nitroanisol. In these products, the strongly deactivating effect of the nitro groups is to large extent compensated by the activating character of the hydroxy- or methoxysubstituent. Thus, the consequent Meerwein arylation with the excess of NBD can proceed to form biphenyl and terphenyl derivatives, which can feature strong adsorption enthalpy to the graphene surface. Formation of such adlayers is then responsible for the emerging $v\left(-\mathrm{NO}_{2}\right)$ and $v(\mathrm{C}=\mathrm{C})$ vibration bands in the Raman spectra.

Without strain - in the particular case of the NBD - the $v\left(-\mathrm{NO}_{2}\right)$ band would be continuously enhancing the apparent $\mathrm{D}$ band intensity, while the $v(\mathrm{C}=\mathrm{C})$ band would appear as an increasing shoulder to the $\mathrm{G}$ band, where, commonly, the intra-valley defect-induced D' peak appears. The average defect distance, $L_{\mathrm{D}}$, can be estimated by the following equation: ${ }^{40}$

$L_{D}^{2}=(1.8 \pm 0.5) \times 10^{-9} \lambda_{L}^{4}\left(\frac{I_{D}}{I_{G}}\right)^{-1}$

where $\lambda_{\mathrm{L}}$ is the Raman excitation wavelength and $\left(I_{\mathrm{D}} / I_{\mathrm{G}}\right)$ the ratio of Raman intensities. We note that eq. 1 is primarily derived for defects created by ion bombardment and the particular multiplier values may differ slightly for other types of defects. However, the dependence of $L_{\mathrm{D}}$ on the $\left(I_{\mathrm{D}} / I_{\mathrm{G}}\right)$ intensity ratio should still follow the same trend. In our case, after $240 \mathrm{~min}$ reaction time, $L_{\mathrm{D}}$ determined from the strain-separated $\mathrm{D}$ and $\mathrm{G}$ peaks yields $\sim 19 \mathrm{~nm}$. If the $\mathrm{D}$ band would not be shifted by strain and thus overlapping with the $v\left(-\mathrm{NO}_{2}\right)$ band, the resulting $L_{\mathrm{D}}$ would amount to $\sim 15.5 \mathrm{~nm}$, i.e. with close to $20 \%$ error.

\section{Conclusions}

In summary, we have shown a simple method to exert biaxial tensile deformation on graphene supported by a polymer (SU8), which is swelling in an appropriate solvent. The attained strain levels were up to $0.7 \%$. In the same time, the experimental procedure can be easily adapted to 
carry out chemical functionalization of graphene by a diazonium salt during the ongoing deformation. Raman spectroscopy was used to monitor the process. The appearance of the D band confirmed the successful functionalization of graphene, however, the simultaneous straininduced downshift of the graphene Raman bands uncovered the presence of additional bands originating from the nitrobenzene diazonium molecule and/or its decomposition products. The intensity evolution of the $\mathrm{D}$ band and of the new bands allowed to discuss the possible reaction mechanism involved. In particular cases, such as the one presented here, attention has to be paid to possible non-graphene Raman bands, which may influence the results of the commonly used quantification of the functionalization degree in graphene.

Supporting Information. Raw (untreated) Raman spectra; additional experiment examples; AFM images, DFT calculation of the Raman vibrations of NBD and its terphenyl derivative.

\section{Acknowledgment}

This work was funded by Czech Science Foundation (GACR 17-18702S). We also acknowledge the support by European Regional Development Fund; OP RDE; Project: "Carbon allotropes with rationalized nanointerfaces and nanolinks for environmental and biomedical applications" (No. CZ.02.1.01/0.0/0.0/16_026/0008382) and the project Pro-NanoEnviCz (Reg. No. CZ.02.1.01/0.0/0.0/16_013/0001821) supported by the Ministry of Education, Youth and Sports of the Czech Republic and the European Union - European Structural and Investments Funds in the frame of Operational Programme Research Development and Education.

\section{References}

1. Novoselov, K. S.; Falko, V. I.; Colombo, L.; Gellert, P. R.; Schwab, M. G.; Kim, K., A Roadmap for Graphene. Nature 2012, 490, 192-200.

2. Li, X. L.; Wang, X. R.; Zhang, L.; Lee, S. W.; Dai, H. J., Chemically Derived, Ultrasmooth Graphene Nanoribbon Semiconductors. Science 2008, 319, 1229-1232.

3. Niyogi, S.; Bekyarova, E.; Itkis, M. E.; Zhang, H.; Shepperd, K.; Hicks, J.; Sprinkle, M.; Berger, C.; Lau, C. N.; deHeer, W. A., et al., Spectroscopy of Covalently Functionalized Graphene. Nano Lett. 2010, 10, 4061-4066. 
4. Levy, N.; Burke, S. A.; Meaker, K. L.; Panlasigui, M.; Zettl, A.; Guinea, F.; Neto, A. H. C.; Crommie, M. F., Strain-Induced Pseudo-Magnetic Fields Greater Than 300 Tesla in Graphene Nanobubbles. Science 2010, 329, 544-547.

5. Bissett, M. A.; Tsuji, M.; Ago, H., Strain Engineering the Properties of Graphene and Other Two-Dimensional Crystals. Phys. Chem. Chem. Phys. 2014, 16, 11124-11138.

6. Park, J.; Yan, M., Covalent Functionalization of Graphene with Reactive Intermediates. Acc. Chem. Res. 2013, 46, 181-189.

7. Huang, P.; Jing, L.; Zhu, H.; Gao, X., Diazonium Functionalized Graphene:

Microstructure, Electric, and Magnetic Properties. Acc. Chem. Res. 2012, 46, 43-52.

8. Bekyarova, E.; Sarkar, S.; Wang, F.; Itkis, M. E.; Kalinina, I.; Tian, X.; Haddon, R. C., Effect of Covalent Chemistry on the Electronic Structure and Properties of Carbon Nanotubes and Graphene. Acc. Chem. Res. 2012, 46, 65-76.

9. Georgakilas, V.; Otyepka, M.; Bourlinos, A. B.; Chandra, V.; Kim, N.; Kemp, K. C.; Hobza, P.; Zboril, R.; Kim, K. S., Functionalization of Graphene: Covalent and Non-Covalent Approaches, Derivatives and Applications. Chem. Rev. 2012, 112, 6156-6214.

10. Paulus, G. L. C.; Wang, Q. H.; Strano, M. S., Covalent Electron Transfer Chemistry of Graphene with Diazonium Salts. Acc. Chem. Res. 2012, 46, 160-170.

11. Nair, R. R.; Ren, W.; Jalil, R.; Riaz, I.; Kravets, V. G.; Britnell, L.; Blake, P.; Schedin, F.; Mayorov, A. S.; Yuan, S., et al., Fluorographene: A Two-Dimensional Counterpart of Teflon. Small 2010, 6, 2877-2884.

12. Kim, G.; Kawazoe, Y.; Lee, K.-R., Controlled Catalytic Properties of Platinum Clusters on Strained Graphene. J. Phys. Chem. Lett. 2012, 3, 1989-1996.

13. Bottari, G.; Herranz, M. Á.; Wibmer, L.; Volland, M.; Rodríguez-Pérez, L.; Guldi, D. M.; Hirsch, A.; Martín, N.; D'Souza, F.; Torres, T., Chemical Functionalization and Characterization of Graphene-Based Materials. Chem. Soc. Rev. 2017, 46, 4464-4500.

14. Koehler, F. M.; Luechinger, N. A.; Ziegler, D.; Athanassiou, E. K.; Grass, R. N.; Rossi, A.; Hierold, C.; Stemmer, A.; Stark, W. J., Permanent Pattern-Resolved Adjustment of the Surface Potential of Graphene-Like Carbon through Chemical Functionalization. Angew. Chem., Int. Ed. 2009, 48, 224-227.

15. Plšek, J.; Kovařriček, P.; Valeš, V.; Kalbáč, M., Tuning the Reactivity of Graphene by Surface Phase Orientation. Chem. Eur. J. 2017, 23, 1839-1845. 
16. Sun, Z.; Pint, C. L.; Marcano, D. C.; Zhang, C.; Yao, J.; Ruan, G.; Yan, Z.; Zhu, Y.; Hauge, R. H.; Tour, J. M., Towards Hybrid Superlattices in Graphene. Nat. Commun. 2011, 2, 559.

17. Wang, Q. H.; Jin, Z.; Kim, K. K.; Hilmer, A. J.; Paulus, G. L. C.; Shih, C.-J.; Ham, M.H.; Sanchez-Yamagishi, J. D.; Watanabe, K.; Taniguchi, T., et al., Understanding and Controlling the Substrate Effect on Graphene Electron-Transfer Chemistry Via Reactivity Imprint Lithography. Nat. Chem. 2012, 4, 724-732.

18. Fan, X.; Nouchi, R.; Tanigaki, K., Effect of Charge Puddles and Ripples on the Chemical Reactivity of Single Layer Graphene Supported by Sio2/Si Substrate. J. Phys. Chem. C 2011, 115, 12960-12964.

19. Bekyarova, E.; Itkis, M. E.; Ramesh, P.; Berger, C.; Sprinkle, M.; de Heer, W. A.; Haddon, R. C., Chemical Modification of Epitaxial Graphene: Spontaneous Grafting of Aryl Groups. J. Am. Chem. Soc. 2009, 131, 1336-1337.

20. Sharma, R.; Baik, J. H.; Perera, C. J.; Strano, M. S., Anomalously Large Reactivity of Single Graphene Layers and Edges toward Electron Transfer Chemistries. Nano Lett. 2010, 10, $398-405$.

21. Lim, H.; Lee, J. S.; Shin, H.-J.; Shin, H. S.; Choi, H. C., Spatially Resolved Spontaneous Reactivity of Diazonium Salt on Edge and Basal Plane of Graphene without Surfactant and Its Doping Effect. Langmuir 2010, 26, 12278-12284.

22. Criado, A.; Melchionna, M.; Marchesan, S.; Prato, M., The Covalent Functionalization of Graphene on Substrates. Angew. Chem., Int. Ed. 2015, 54, 10734-10750.

23. Koehler, F. M.; Jacobsen, A.; Ihn, T.; Ensslin, K.; Stark, W. J., Chemical Modification of Graphene Characterized by Raman and Transport Experiments. Nanoscale 2012, 4, 3781-3785.

24. Koehler, F. M.; Jacobsen, A.; Ensslin, K.; Stampfer, C.; Stark, W. J., Selective Chemical Modification of Graphene Surfaces: Distinction between Single- and Bilayer Graphene. Small 2010, 6, 1125-1130.

25. Menanteau, T.; Dias, M.; Levillain, E.; Downard, A. J.; Breton, T., Electrografting Via Diazonium Chemistry: The Key Role of the Aryl Substituent in the Layer Growth Mechanism. $J$. Phys. Chem. C 2016, 120, 4423-4429. 
26. Mueller, N. S.; Heeg, S.; Peña-Alvarez, M.; Kusch, P.; Wasserroth, S.; Clark, N.; Schedin, F.; Parthenios, J.; Papagelis, K.; Galiotis, C., et al., Evaluating Arbitrary Strain Configurations and Doping in Graphene with Raman Spectroscopy. 2D Mater. 2018, 5, 015016.

27. Lee, J. E.; Ahn, G.; Shim, J.; Lee, Y. S.; Ryu, S., Optical Separation of Mechanical Strain from Charge Doping in Graphene. Nat. Commun. 2012, 3, 1024.

28. Androulidakis, C.; Koukaras, E. N.; Parthenios, J.; Kalosakas, G.; Papagelis, K.; Galiotis, C., Graphene Flakes under Controlled Biaxial Deformation. Sci. Rep. 2015, 5, 18219.

29. Zabel, J.; Nair, R. R.; Ott, A.; Georgiou, T.; Geim, A. K.; Novoselov, K. S.; Casiraghi, C., Raman Spectroscopy of Graphene and Bilayer under Biaxial Strain: Bubbles and Balloons. Nano Lett. 2012, 12, 617-621.

30. Gong, L.; Kinloch, I. A.; Young, R. J.; Riaz, I.; Jalil, R.; Novoselov, K. S., Interfacial Stress Transfer in a Graphene Monolayer Nanocomposite. Adv. Mater. 2010, 22, 2694-2697.

31. Anagnostopoulos, G.; Androulidakis, C.; Koukaras, E. N.; Tsoukleri, G.; Polyzos, I.; Parthenios, J.; Papagelis, K.; Galiotis, C., Stress Transfer Mechanisms at the Submicron Level for Graphene/Polymer Systems. ACS Appl. Mater. Interfaces 2015, 7, 4216-4223.

32. Androulidakis, C.; Koukaras, E. N.; Frank, O.; Tsoukleri, G.; Sfyris, D.; Parthenios, J.; Pugno, N.; Papagelis, K.; Novoselov, K. S.; Galiotis, C., Failure Processes in Embedded Monolayer Graphene under Axial Compression. Sci. Rep. 2014, 4, 5271.

33. Wouters, K.; Puers, R., Diffusing and Swelling in Su-8: Insight in Material Properties and Processing. J. Micromech. Microeng. 2010, 20, 095013.

34. Malard, L. M.; Pimenta, M. A.; Dresselhaus, G.; Dresselhaus, M. S., Raman Spectroscopy in Graphene. Phys. Rep. 2009, 473, 51-87.

35. Vandenabeele, P.; Moens, L.; Edwards, H. G. M.; Dams, R., Raman Spectroscopic Database of Azo Pigments and Application to Modern Art Studies. J. Raman Spectrosc. 2000, $31,509-517$.

36. Kovaříček, P.; Vrkoslav, V.; Plšek, J.; Bastl, Z.; Fridrichová, M.; Drogowska, K.; Kalbáč, M., Extended Characterization Methods for Covalent Functionalization of Graphene on Copper. Carbon 2017, 118, 200-207.

37. Bouša, D.; Jankovský, O.; Sedmidubský, D.; Luxa, J.; Šturala, J.; Pumera, M.; Sofer, Z., Mesomeric Effects of Graphene Modified with Diazonium Salts: Substituent Type and Position Influence Its Properties. Chem. Eur. J. 2015, 21, 17728-17738. 
38. Lomeda, J. R.; Doyle, C. D.; Kosynkin, D. V.; Hwang, W.-F.; Tour, J. M., Diazonium Functionalization of Surfactant-Wrapped Chemically Converted Graphene Sheets. J. Am. Chem. Soc. 2008, 130, 16201-16206.

39. Kasprzak, A.; Zuchowska, A.; Poplawska, M., Functionalization of Graphene: Does the Organic Chemistry Matter? Beilstein J. Org. Chem. 2018, 14, 2018-2026.

40. Cançado, L. G.; Jorio, A.; Ferreira, E. H. M.; Stavale, F.; Achete, C. A.; Capaz, R. B.; Moutinho, M. V. O.; Lombardo, A.; Kulmala, T. S.; Ferrari, A. C., Quantifying Defects in Graphene Via Raman Spectroscopy at Different Excitation Energies. Nano Lett. 2011, 11, 31903196.

\section{TOC Graphic}

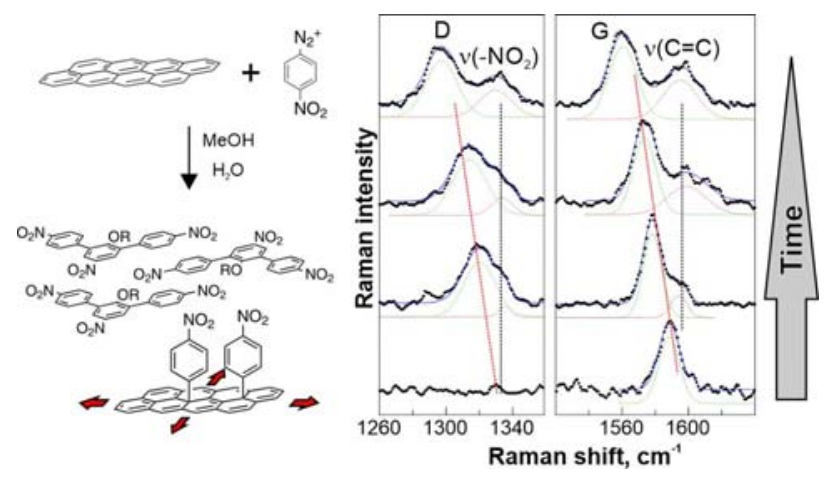




\section{Supporting Information}

\section{On the Suitability of Raman Spectroscopy to Monitor the Degree of Graphene Functionalization by Diazonium Salts}

Krishna Sampathkumar ${ }^{1,2}$, Valentin Diez-Cabanes ${ }^{1,3 \#}$, Petr Kovaricek ${ }^{1}$, Elena del Corro ${ }^{1, \$}$, Milan Bouša ${ }^{1}$, Jan Hošek ${ }^{3}$, Martin Kalbac ${ }^{1}$, Otakar Frank ${ }^{1, *}$

${ }^{1}$ J.Heyrovsky Institute of Physical Chemistry of the CAS, v.v.i., Dolejskova 2155/3, 18200

Praha 8, Czech Republic

${ }^{2}$ Central European Institute of Technology (CEITEC), Brno University of Technology, Purkyňova 123, 61200 Brno, Czech Republic

${ }^{3}$ Faculty of Mechanical Engineering, Czech Technical University in Prague, Technická 4, 16 607 Prague 6, Czech Republic

\# current address: Laboratory for Chemistry of Novel Materials, University of Mons, Place du Parc 20, B-7000, Mons, Belgium.

\$ current address: Catalan Institute of Nanoscience and Nanotechnology (ICN2), CSIC and the Barcelona Institute of Science and Technology (BIST), Barcelona, Spain.

\section{Corresponding Author}

* otakar.frank@jh-inst.cas.cz, tel: +420 266053446 


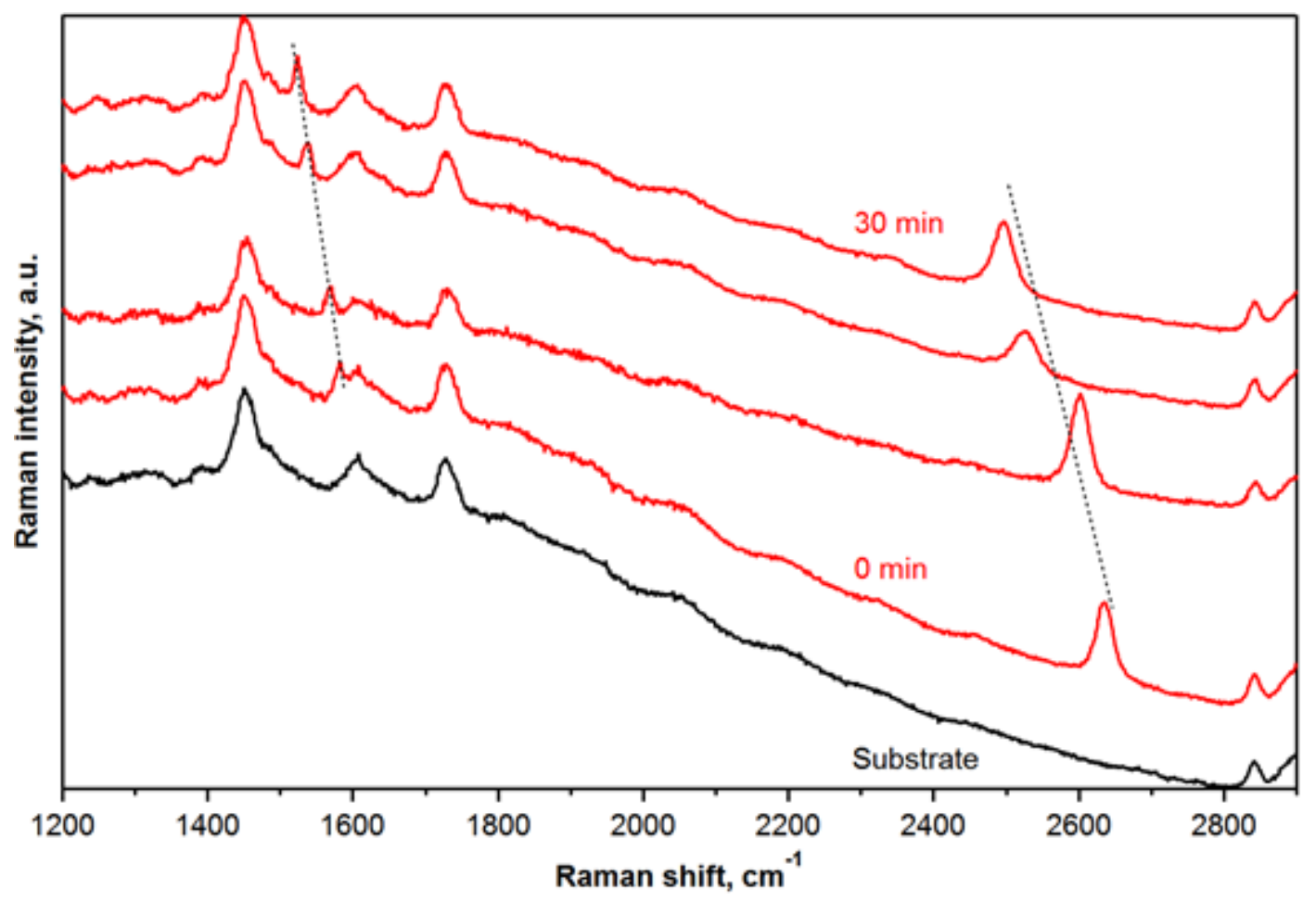

Figure S1. Raw Raman spectra of monolayer graphene (red) on PMMA-SU8 substrate (black) dipped in 1:1 methanol:water solution for increasing time, measured in the approximate center of the flake. The downshifting $\mathrm{G}$ and $2 \mathrm{D}$ bands are indicated by dashed lines.
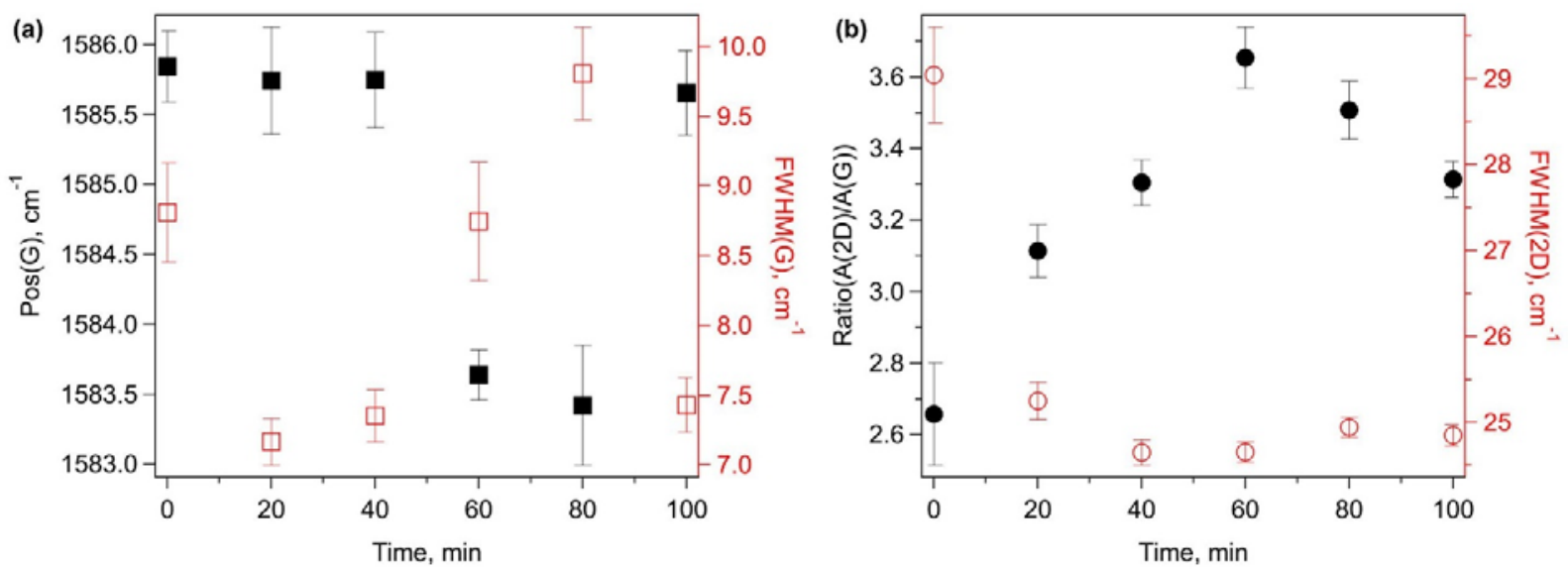

Figure S2. Blank experiment of $1 \mathrm{~L}$ graphene on $\mathrm{Si} / \mathrm{SiO}_{2}(300 \mathrm{~nm})$ substrate immersed in methanol:water (1:1) solution for an indicated time. Evolution of (a) the Raman G band (shift - left axis, FWHM - right axis) and (b) area(2D/G) ratio (left axis) and FWHM(2D) (right axis), shows only minor fluctuations in part due to inhomogeneities across the flake and in part due to doping originating probably from glue remnants from the exfoliation. The data points represent average from 9 measurements, with the error bars the standard deviation. 

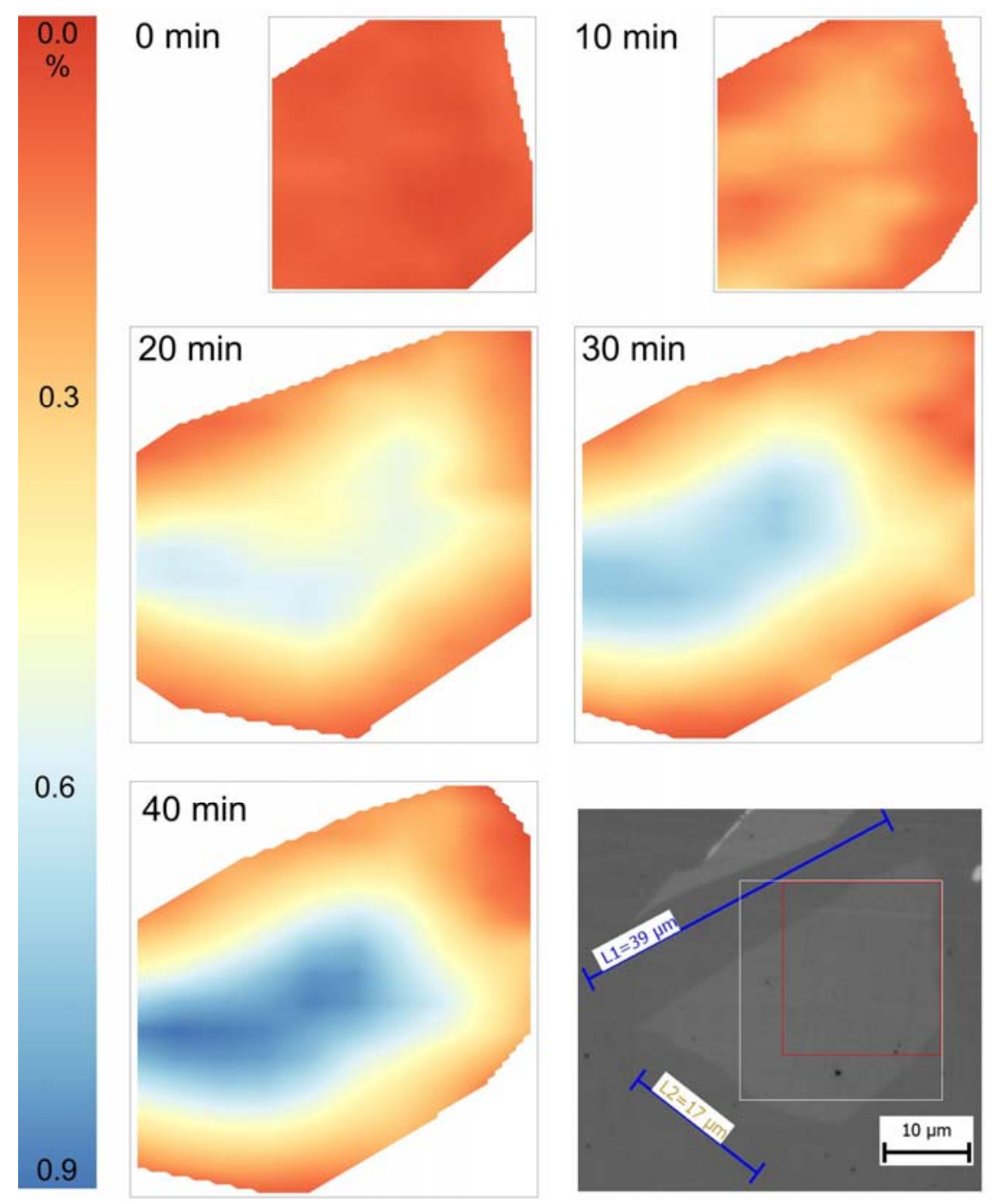

Figure S3. Evolution of the Raman spectral features of monolayer graphene on PMMA/SU8 substrate subjected to swelling in methanol:water solution. Maps of biaxial strain quantified from the $2 \mathrm{D}$ band shift at $0,10,20,30$ and 40 minutes of soaking and optical image of the flake with the mapped area at 0 and 10 minutes (marked red), and 20, 30 and 40 minutes (white). The mapping step was $2 \mu \mathrm{m}$ in each direction. 

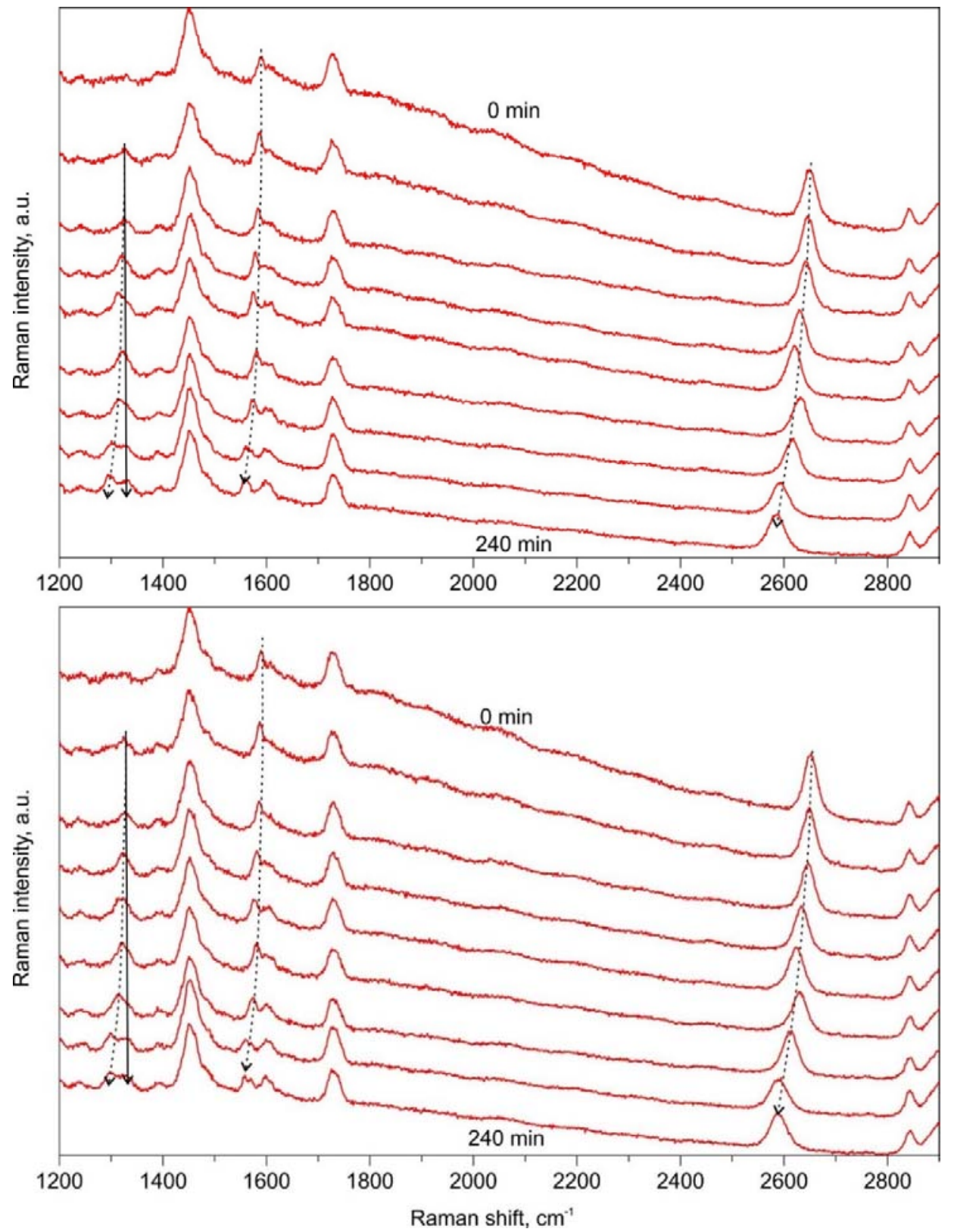

Figure S4. Evolution of Raman spectra measured at two different spots of monolayer graphene on SU8/PMMA treated with $0.01 \mathrm{M}$ solution (1:1 water:methanol) of 4-nitrobenzene diazonium tetrafluoroborate for 0 to 240 minutes (from top to bottom). The D, G and 2D band are marked by dashed black lines (from left to right), the $v\left(-\mathrm{NO}_{3}\right)$ vibration by solid black line. The spectra in the top panel were acquired in the approximate center of the flake, the spectra in the bottom panel approximately at half the distance between the center and the edge of the flake. 

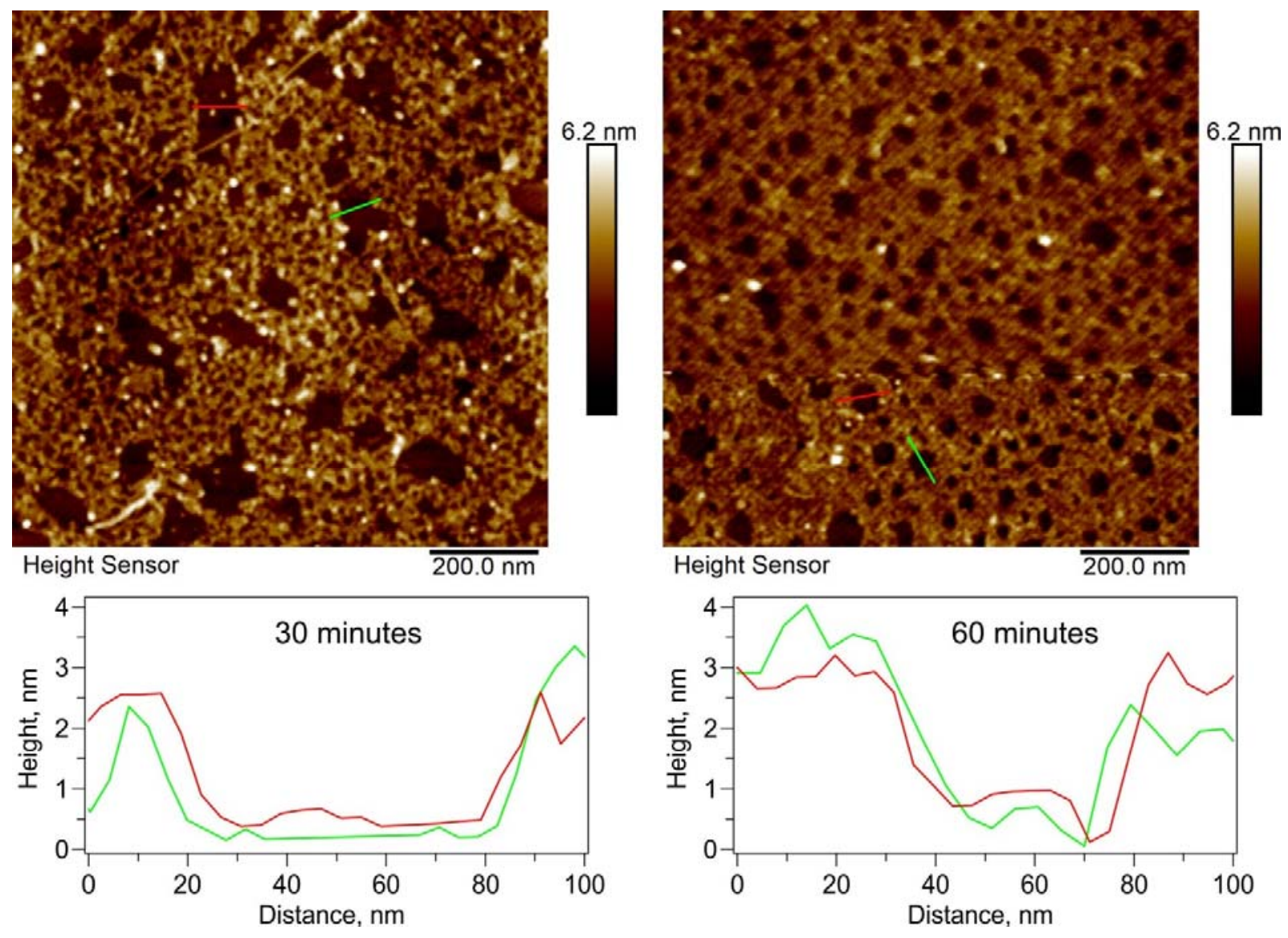

Figure S5. AFM images and extracted height profiles of monolayer graphene on SU8/PMMA treated with $0.01 \mathrm{M}$ solution (1:1 water:methanol) of 4-nitrobenzene diazonium tetrafluoroborate for 30 minutes (left) and 60 minutes (right). The total covered area increases from 53 to $81 \%$, i.e. by a factor of $\sim 1.5$. The average height difference between the covered and uncovered area is the same in both cases: $1.4 \mathrm{~nm}$.

\section{Theoretical section}

Geometrical optimizations of 4-nitrobenzene diazonium compound (1) and its terphenyl derivative

(2) (see Figure S6) were carried out at Density Functional Theory (DFT) level within the Becke, 3-parameter, Lee-Yang-Parr (B3LYP) functional ${ }^{1}$ and 6-311G(d,p) basis set. The calculation of the Raman spectra was done with the same functional and basis set. In the case of the terphenyl reaction product, the vibrational properties of this compound are independent of the group attached 
to the central phenyl ring: hydroxy- vs methoxy- substituent (see Figure S7). For that reason, in the following discussion we consider only the first case.

1)<smiles>O=[N+]([O-])c1ccccc1</smiles><smiles>[R]Oc1c(-c2ccc([N+](=O)[O-])cc2)cc([N+](=O)[O-])cc1-c1ccc([N+](=O)[O-])cc1</smiles>

Figure S6. Chemical structures of the compounds under study.

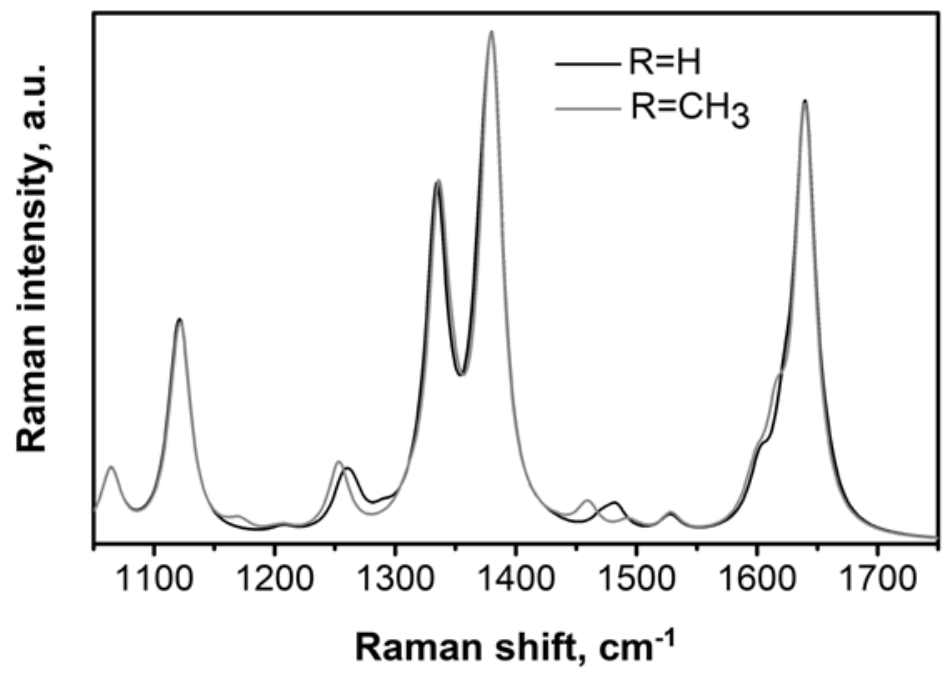

Figure S7. Calculated Raman spectra of compound 2 with hydroxy- (grey) and methoxy- (black line) substituents on the central rings as depicted in Figure S6. Note that the range of frequencies plotted (1050-1750 $\left.\mathrm{cm}^{-1}\right)$ has been chosen in order to show the modes which are overlapping the $\mathrm{G}$ and $\mathrm{D}$ bands from garphene, whereas no important contributions to the spectra were found in the range of frequencies for graphene 2D band.

The solvent effects were introduced into our calculations by means of the Polarizable Continuum Model (PCM). ${ }^{2}$ The calculated Raman spectra for both methanol and water solvents are identical, while a shift of $\sim 15 \mathrm{~cm}^{-1}$ is induced when adding the solvent effects compared to the spectra calculated in the gas phase for both compounds (see Figure S8). This fact evidences the need of 
including solvent effects for a proper description of the normal modes frequencies. All theoretical calculations were performed within Gaussian 16 package program, ${ }^{3}$ whereas the Raman intensities were estimated for an excitation wavelength of $\lambda=633 \mathrm{~nm}$ and temperature $\mathrm{T}=300 \mathrm{~K}$ by using GaussSum 3.0 plot program. ${ }^{4}$

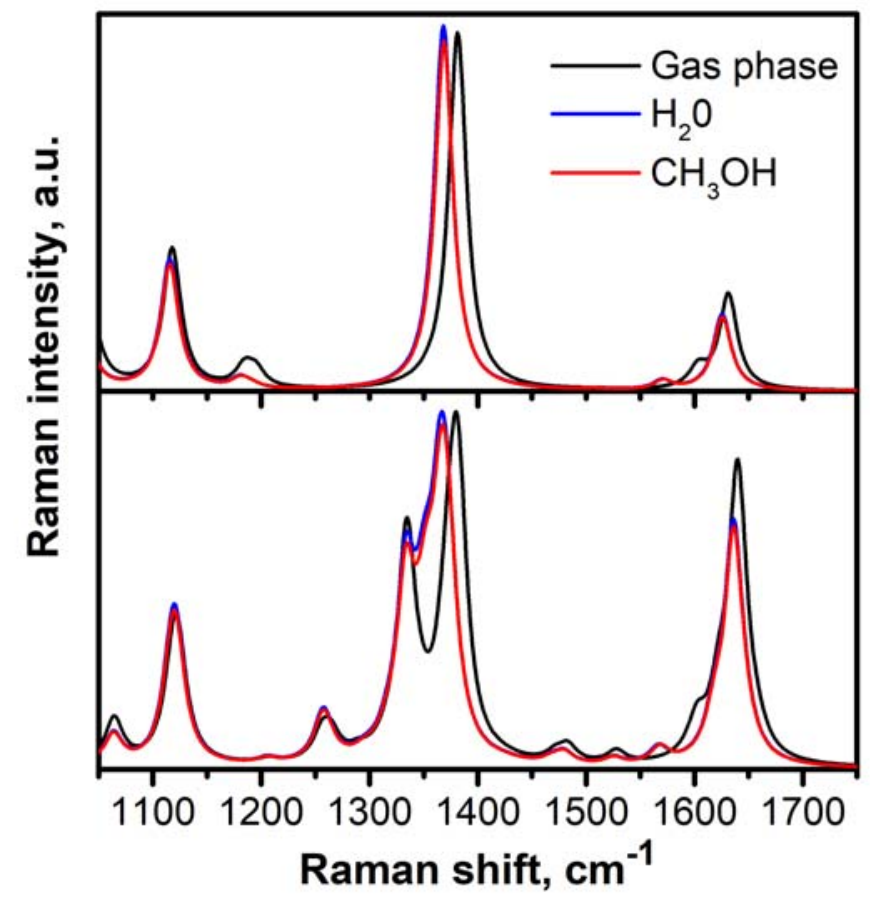

Figure S8. Calculated Raman spectra for compounds 1 (top) and 2 (bottom) for the calculations performed in gas phase (black), water (blue) and methanol (red) solvents.

The calculated Raman spectra for compounds $\mathbf{1}$ and $\mathbf{2}$ are presented in Figure S9. The spectrum of compound 1 in the region of interest is dominated by two normal modes centered at 1368 and 1625 $\mathrm{cm}^{-1}$. If we compare this spectrum with the band signatures which are appearing in the measured spectra (Fig. 3, main text) as a consequence of the functionalization with NBD, we can easily attribute the above mentioned calculated modes to the $v\left(-\mathrm{NO}_{2}\right)$ and $v(\mathrm{C}=\mathrm{C})$ vibration bands described in the main text. The small discrepancies in the absolute frequencies (the calculated spectra are $\sim 25 \mathrm{~cm}^{-1}$ red-shifted with respect to the experimental frequencies) can be attributed to 
the facts that (i) neither the interactions with the polymer/graphene substrate nor with the neighboring molecules were included in our theoretical model and (ii) the measurement was performed on dry samples (the calculation in a solvent, which has an obvious effect on the frequencies, see above). On the other hand, the calculated spectrum of compound 2 presents the same features as the spectrum from compound $\mathbf{1}$. In this case, the normal modes mentioned before are degenerated due to the effect of two additional nitrobenzene groups, whereas a new normal mode is appearing at $1332 \mathrm{~cm}^{-1}$ close to the bands attributed to the $v\left(-\mathrm{NO}_{2}\right)$ signatures.

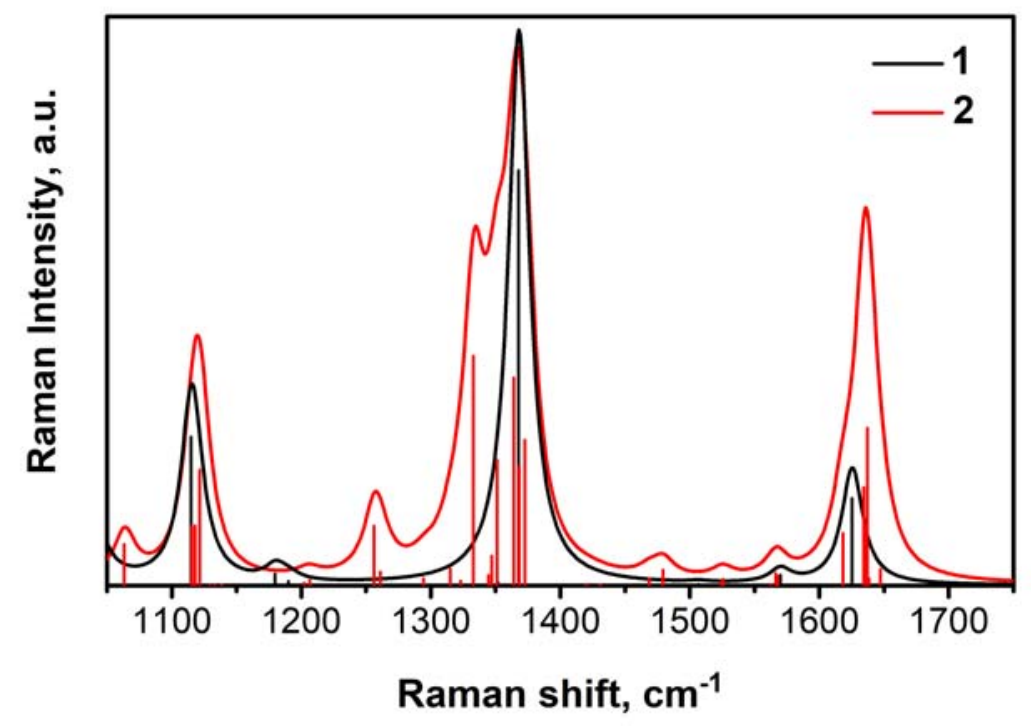

Figure S9. Calculated Raman spectra for compounds 1 (top) and 2 (bottom) in water solvent. The vertical bars represent the normalized amplitude of the Raman intensities for each normal mode.

In order to get a deeper understanding of the vibrational modes attributed to the $v\left(-\mathrm{NO}_{2}\right)$ and $v(\mathrm{C}=\mathrm{C})$ bands, we have plotted the normalized force vectors for the calculated normal modes described in the previous paragraph (see Figure S10). The vibrational mode of $v\left(-\mathrm{NO}_{2}\right)$ in compound $\mathbf{1}$ is based on the stretching mode of $\mathrm{C}-\mathrm{N}$ bond for the nitro- group, which is centered at $1368 \mathrm{~cm}^{-1}$. The same stretching mode is also dominating the spectra of compound $\mathbf{2}$, in this case centered at $1364 \mathrm{~cm}^{-1}$. The rest of contributions to the $v\left(-\mathrm{NO}_{2}\right)$ band in compound 2 are attributed 
to the stretching vibration of the N-O atoms from the nitro- group as it is the case for the normal modes centered at 1351 and $1368 \mathrm{~cm}^{-1}$; and to the bending vibration of the methoxy- group which dominates the normal mode centered at $1372 \mathrm{~cm}^{-1}$. In the case of the band $v(\mathrm{C}=\mathrm{C})$ band, the $\mathrm{E}_{2 \mathrm{~g}}$ mode of the benzene core is the dominant vibration for both compounds $\mathbf{1}$ and $\mathbf{2}$. Interestingly, for compound 2 the edge phenyl ring vibrations (centered at 1634, 1635 and $1637 \mathrm{~cm}^{-1}$ ) present larger Raman intensities than the central ring mode (centered at $1618 \mathrm{~cm}^{-1}$ ). Finally, we have analyzed the force vectors of normal mode which is appearing at $1332 \mathrm{~cm}^{-1}$ in compound 2 (Figure $\mathrm{S} 11$ ). This vibration is a combination of several modes of the benzene cores, from which we can highlight the $\mathrm{A}_{2 \mathrm{~g}}$ mode of the edge phenyl rings. 


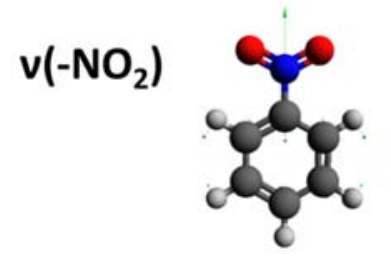

$v=1368 \mathrm{~cm}^{-1}(32836)$

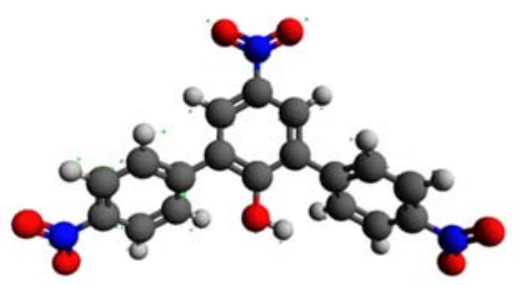

$\mathrm{v}=1351 \mathrm{~cm}^{-1}(66172)$

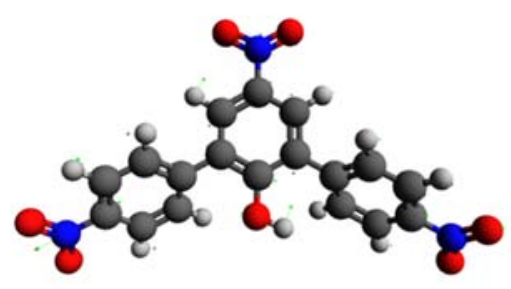

$v=1364 \mathrm{~cm}^{-1}(109654)$

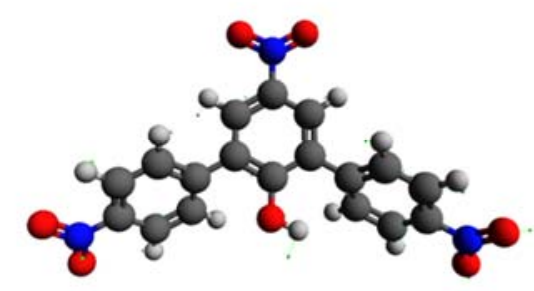

$v=1368 \mathrm{~cm}^{-1}(62784)$

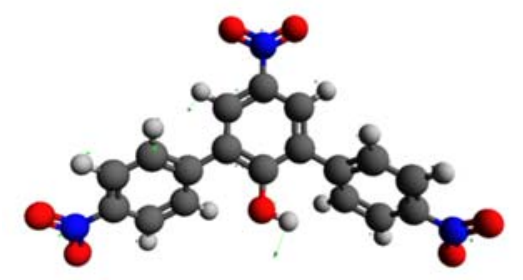

$v=1372 \mathrm{~cm}^{-1}(76628)$

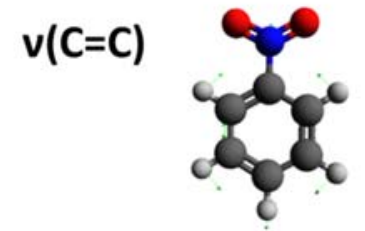

$v=1625 \mathrm{~cm}^{-1}(6889)$

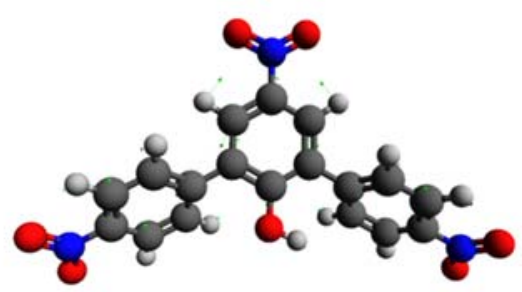

$\mathrm{v}=1618 \mathrm{~cm}^{-1}$ (27519)

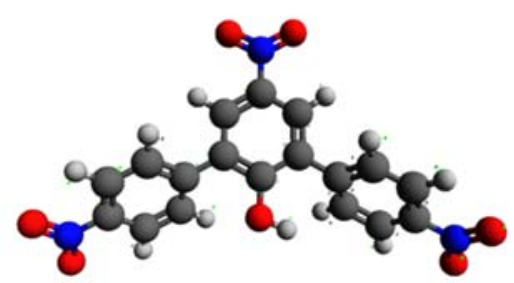

$\mathrm{v}=1634 \mathrm{~cm}^{-1}(51749)$

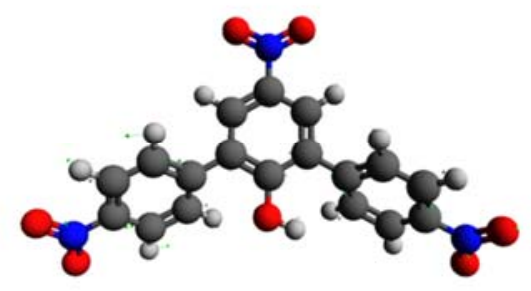

$\mathrm{v}=1635 \mathrm{~cm}^{-1}(32065)$

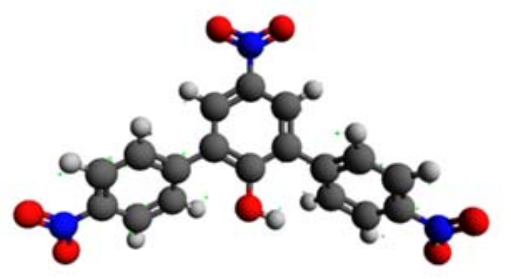

$v=1637 \mathrm{~cm}^{-1}(82931)$

Figure S10. Normalized force vectors of the vibrational modes attributed to the $v\left(-\mathrm{NO}_{2}\right)$ and $v(\mathrm{C}=\mathrm{C})$ bands for compounds $\mathbf{1}$ (top) and $\mathbf{2}$ (bottom). The numbers inset represent the frequencies and Raman intensities (in a.u. and parenthesis) for each vibrational mode. 


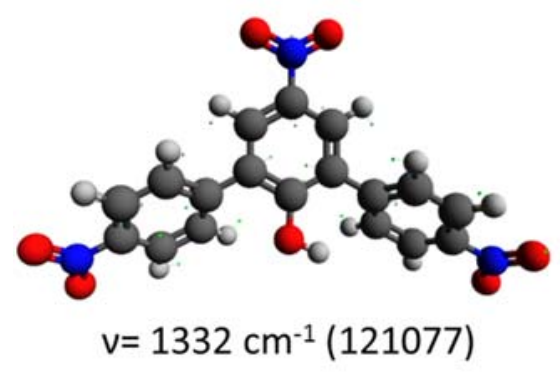

Figure S11. Normalized force vectors of the vibrational mode appearing at $1332 \mathrm{~cm}^{-1}$ for compound 2. The numbers inset represent the frequencies and Raman intensities (in a.u. and parenthesis).

\section{References}

(1) Becke, A. D. Density-functional thermochemistry. III. The role of exact exchange. J. Chem. Phys. 98, 5648-5652 (1993).

(2) Tomasi, J.; Mennucci, B.; \& Cammi, R. Quantum mechanical continuum solvation models, Chem. Rev. 105, 2999-3093 (2015).

(3) Frisch, M. J.; Trucks, G. W.; Schlegel, H. B.; Scuseria, G. E.; Robb, M. A.; Cheeseman, J. R.; Scalmani, G.; Barone, V.; Petersson, G. A.; Nakatsuji, H.; Li, X.; Caricato, M.; Marenich, A. V.; Bloino, J.; Janesko, B. G.; Gomperts, R.; Mennucci, B.; Hratchian, H. P.; Ortiz, J. V.; Izmaylov, A. F.; Sonnenberg, J. L.; Williams-Young, D.; Ding, F.; Lipparini, F.; Egidi, F.; Goings, J.; Peng, B.; Petrone, A.; Henderson, T.; Ranasinghe, D.; Zakrzewski, V. G.; Gao, J.; Rega, N.; Zheng, G.; Liang, W.; Hada, M.; Ehara, M.; Toyota, K.; Fukuda, R.; Hasegawa, J.; Ishida, M.; Nakajima, T.; Honda, Y.; Kitao, O.; Nakai, H.; Vreven, T.; Throssell, K.; Montgomery, J. A., Jr.; Peralta, J. E.; Ogliaro, F.; Bearpark, M. J.; Heyd, J. J.; Brothers, E. N.; Kudin, K. N.; Staroverov, V. N.; Keith, T. A.; Kobayashi, R.; Normand, J.; Raghavachari, K.; Rendell, A. P.; Burant, J. C.; Iyengar, S. S.; Tomasi, J.; Cossi, M.; Millam, J. M.; Klene, M.; Adamo, C.; Cammi, R.; Ochterski, J. W.; Martin, R. L.; Morokuma, K.; Farkas, O.; Foresman, J. B.; \& Fox, D. J. Gaussian 16, Revision B.01, Gaussian, Inc., Wallingford CT (2016).

(4) O'Boyle, N. M.; Tenderholt, L. A. \& Langner, K. M. cclib: A library for packageindependent computational chemistry algorithms. J. Comput. Chem. 29, 839-845 (2008). 